\title{
Impacts of an opioid overdose prevention intervention delivered subsequent to acute care
}

\author{
Caleb J Banta-Green, ${ }^{1}$ Phillip 0 Coffin, ${ }^{2,3}$ Joseph 0 Merrill, ${ }^{4}$ Jeanne M Sears, ${ }^{5,6}$ \\ Chris Dunn, ${ }^{7}$ Anthony S Floyd, ${ }^{8}$ Lauren K Whiteside, ${ }^{9}$ Norbert D Yanez, ${ }^{10}$ \\ Dennis M Donovan ${ }^{11}$
}

Additional material is published online only. To view please visit the journal online (http://dx.doi.org/10.1136/ injuryprev-2017-042676)

For numbered affiliations see end of article.

Correspondence to Dr Caleb J Banta-Green, Alcohol \& Drug Abuse Institute and Department of Health Services, School of Public Health, University of Washington, Seattle, WA 98105, USA; calebbg@u.washington.edu

Received 25 November 2017 Revised 16 January 2018 Accepted 18 January 2018 Published Online First 7 February 2018

\begin{abstract}
Background Opioid overdose is a major and increasing cause of injury and death. There is an urgent need for interventions to reduce overdose events among high-risk persons.

Methods Adults at elevated risk for opioid overdose involving heroin or pharmaceutical opioids who had been cared for in an emergency department (ED) were randomised to overdose education combined with a brief behavioural intervention and take-home naloxone or usual care. Outcomes included: (1) time to first opioid overdose-related event resulting in medical attention or death using competing risks survival analysis; and (2) ED visit and hospitalisation rates, using negative binomial regression and adjusting for time at risk.
\end{abstract}

Results During the follow-up period, 24\% of the 241 participants had at least one overdose event, $85 \%$ had one or more ED visits and $55 \%$ had at least one hospitalisation, with no significant differences between intervention and comparison groups. The instantaneous risk of an overdose event was not significantly lower for the intervention group (sub-HR: $0.83 ; 95 \% \mathrm{Cl} 0.49$ to 1.40).

Discussion These null findings may be due in part to the severity of the population in terms of housing insecurity (70\% impermanently housed), drug use, unemployment and acute healthcare issues. Given the high overdose and healthcare utilisation rates, more intensive interventions, such as direct referral and provision of housing and opioid agonist treatment medications, may be necessary to have a substantial impact on opioid overdoses for this high-acuity population in acute care settings.

Trial registration number NCT0178830; Results.

\section{INTRODUCTION}

Opioid overdose deaths continue to increase and are a major cause of preventable death. ${ }^{1}$ According to the CDC, opioid-involved overdose deaths quadrupled from 1999 to 2015 when there were 33091 such deaths in the USA. ${ }^{2}$ Additionally, opioid-related emergency department (ED) visits and inpatient admissions have increased dramatically over the years reflecting the increase in non-fatal overdose. ${ }^{3}$ Many overdoses are amenable to intervention due to biological and social circumstances. ${ }^{4}$ Opioid overdoses rarely lead to sudden death, with death usually occurring several hours after consumption, ${ }^{5}$ though this may be changing as illicit synthetic opioids with their rapid rates of onset and high potency emerge as major causes of death. ${ }^{6}$ Moreover, most overdoses occur in the presence of another person, ${ }^{78}$ providing an opportunity for bystander intervention.

Brief behaviour change counselling is based on motivational interviewing (MI), ${ }^{9}$ has been found to help reduce drug use frequency ${ }^{10}$ and to significantly improve health behaviours such as alcohol use and injury, to increase entry into drug abuse treatment and to reduce costs in ED. ${ }^{11} 12$ In pharmaceutical opioid using patients in the ED with elevated risk for overdose, patients receiving a brief behavioural intervention had decreased overdose risk behaviours. ${ }^{13}$ Additionally, brief intervention has been used to decrease drug use among patients in the ED. ${ }^{14}$ However, these studies did not specifically target illicit opioid use and did not combine brief behaviour change counselling with takehome naloxone. We combined these interventions based on the information-motivation-behaviour model, positing that overdose education, combined with self-identified motivating factors and the behavioural skills to utilise naloxone, might impact overdose occurrence. ${ }^{15}$

Naloxone is an opioid-antagonist prescription medication that reverses opioid overdoses by preferentially binding to opioid receptors and displacing opioids such as heroin, morphine, oxycodone and fentanyl and reversing respiratory depression and sedation. Naloxone cannot be abused, has no psychoactive effects and has been found to be extremely safe. ${ }^{1617}$ Since the 1990 s, naloxone has increasingly been provided to people who use drugs through low-threshold service programmes such that by 2014 community-based programmes were distributing naloxone to laypersons at 644 sites in the USA. ${ }^{18}$ Take-home naloxone for lay people has been recommended by organisations such as the WHO and in the Substance Abuse and Mental Health Services Administration opioid overdose toolkit. $^{1920}$

Research on take-home naloxone provided to people at risk for having or witnessing an overdose indicates that: (1) naloxone administration has not resulted in dangerous health consequences ${ }^{21}$; (2) lay persons can be trained to recognise an overdose and evaluate whether administration of naloxone is warranted as well as medical experts ${ }^{22}$; (3) illicit drug users are willing to administer naloxone to each other ${ }^{23}$; (4) naloxone availability does not increase drug use ${ }^{24}$; (5) many opioid overdoses have been reversed with naloxone as a result of 
overdose prevention and recognition training combined with the distribution of take-home naloxone $\mathrm{e}^{25}$; and (6) provision of naloxone is associated with reduced mortality in communities that implement the programme compared with communities that do not and reduced opioid overdose mortality after release from prison. ${ }^{25} 26$ However, there are no trials that aim to assess the impact of pairing naloxone provision with a brief behavioural intervention in healthcare settings on subsequent overdose events and healthcare utilisation.

ED and acute care settings are potentially advantageous settings to reach populations vulnerable to opioid overdose that may not access healthcare in other settings such as substance use disorder treatment centres or primary care. In this study, we tested an intervention for opioid users at elevated risk for overdose that was delivered during or after an acute care episode that combined opioid overdose education, a take-home naloxone kit and brief behaviour change counselling to determine the impact on participants' subsequent opioid overdoses, ED visits and hospitalisations.

\section{METHODS}

\section{Setting}

Participants were enrolled subsequent to a visit at one of two EDs in Seattle: Harborview Medical Center (HMC) and the University of Washington Medical Center (UWMC). HMC is a large urban academic medical centre dedicated to an underserved population including those with mental health and substance use problems. UWMC is an academic tertiary care hospital providing extensive specialty care. Potential participants were identified through medical records review by study staff or healthcare staff referrals. Recruitment and enrolment generally occurred between the hours of 12:00 and 20:00 weekdays in the $\mathrm{ED}$, in other hospital units during subsequent inpatient admission or at respite care (a recuperative care facility adjacent to HMC for homeless people who require medical assistance and shelter and do not require inpatient treatment).

\section{Participants}

Potential participants were identified either by study staff reviewing electronic medical records or by medical staff referral with eligibility confirmed via a screening questionnaire. Eligibility criteria included being at elevated risk of opioid overdose based on: (1) reason for visit was opioid overdose; (2) use of pharmaceutical opioids not prescribed two or more times in the prior month; (3) use of other opioids, alcohol, sedatives or stimulants within 2 hours of using opioids two or more times in the prior month; (4) average daily dose of prescribed opioids greater than $10 \mathrm{mg}$ morphine equivalent dose or higher for 15 or more of the last 30 days; or (5) enrolled in an opioid agonist therapy (OAT) programme and receiving methadone or buprenorphine. Opioids needed to be used at least twice in the last 30 days (or if institutionalised recently, in the most recent month they were not institutionalised) with pharmaceutical users also needing to have other risks present. Subjects were not excluded if pregnant and were offered naloxone if in the intervention arm and informed during consent about potential risks to a fetus due to precipitated withdrawal and the need to seek emergency medical care.

Exclusion criteria included: (1) refusing access to follow-up medical or drug treatment records; (2) inability to communicate in English; (3) current suicidal ideation; (4) significant cognitive or psychiatric impairment; (5) inability to provide adequate contact information to assist with follow-up (the number of required contacts was reduced after a month of recruitment from three to one as most homeless people were being excluded that would have negatively impacted the generalisability of the findings); (6) under age 18 years or over age 70 years; (7) not living in Washington State or planning to move from Washington State within a year; (8) receiving treatment for sexual assault; or (9) currently having non-expired naloxone.

Potential participants provided informed consent for eligibility screening. If eligible and interested in the study, consent was obtained for study participation. Eligibility screening and study participation were remunerated with $\$ 5$ and $\$ 20$ store gift cards, respectively. Follow-up surveys were conducted at 3, 6 and 12 months (with $\$ 10, \$ 10$ and $\$ 20$ gift card remunerations, respectively); these data are not presented here as follow-up rates were below $50 \%$ at each time point. Releases of information and HIPAA (Health Insurance Portability and Accountability Act) authorisations were obtained to access medical records and drug treatment data. Baseline data were collected by interventionists in clinical settings, with an attempt to maximise privacy, and participants were randomised; this process took approximately 30-45 min. An unrestricted or 'fair-coin' randomisation process was used to generate a study assignment table based on study identification numbers and implemented automatically via Research Electronic Data Capture (REDCap) with interventionists learning study assignment at the same time as the participant.

Study data were collected and managed using REDCap electronic data capture, secure web-based tools hosted at the Institute of Translational Health Sciences at the University of Washington.

\section{Intervention}

The intervention consisted of (1) overdose education, (2) a brief behavioural change counselling component to assist participants in identifying their overdose risks and the steps they were interested in taking to reduce those risks and (3) a naloxone kit. The intervention was provided by two interventionists who had master's degree and at least basic training in MI.

Overdose education included watching an $8 \mathrm{~min}$ video and reviewing an informational flier with the interventionist, which addressed risk factors for an opioid overdose, overdose recognition, recommendation to call 911, how to administer naloxone and guidance to remain with the overdose victim for several hours. The flier also provided specific information about locations where naloxone could be obtained either free at area syringe exchange programmes or for purchase at a local pharmacy. The flier included a link to www.stopoverdose.org, which has online overdose educational materials, including the training video used at the time created by the New York City Department of Health as well as a naloxone locator for Washington State.

Naloxone administration training included hands-on practice assembling the kit,which included a luer lock syringe, $2 \mathrm{mg} / 2 \mathrm{~mL}$ naloxone (Amphastar NDC\#76329-3369-1) and a mucosal atomisation device. Intranasal administration was an off-label route of administration, and a Food and Drug Administration Investigational New Drug application was required (\#112 043). The kit included two doses of naloxone, two mucosal atomisers, a disposable rescue breathing mask, a wallet card with information about Washington State's Good Samaritan Overdose and naloxone access law (RCW 69.50.315) and the educational flier all packaged in a nylon pouch. Participants were directly handed the kit by study staff; however, they did not need to accept the offer of the kit to be considered a study participant.

Participants assigned to the comparison group were provided the informational flier. 


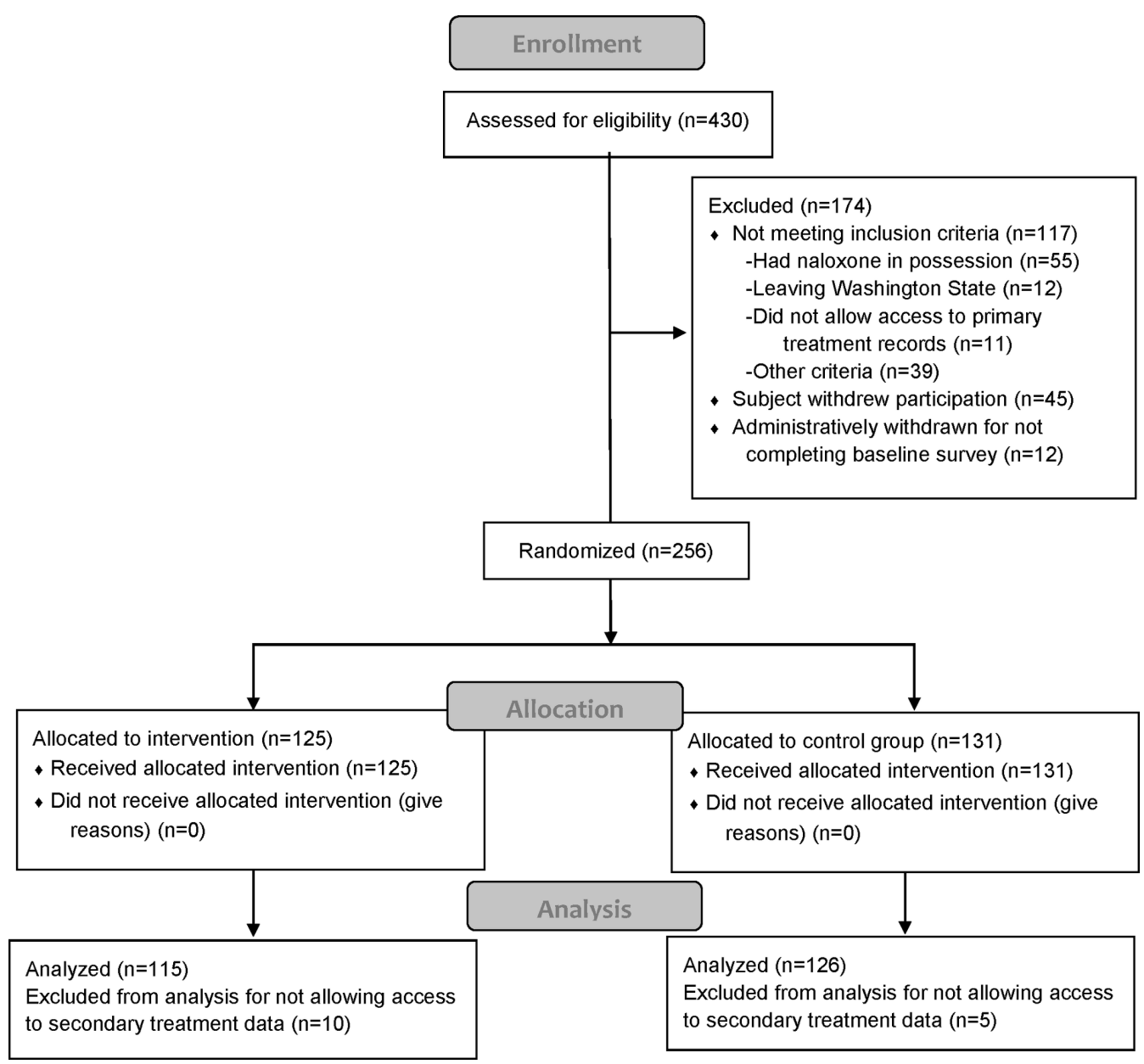

Figure 1 CONSORT diagram, study enrolment, allocation and analysis. CONSORT, Consolidated Standards of Reporting Trials.

\section{Intervention fidelity}

Sessions were audio-recorded, and initial training and regular supervision with a doctoral level psychologist (CD) occurred throughout the study. A sample of intervention recordings were reviewed for fidelity. The intervention was MI)inspired in order to facilitate rapport building and participant engagement. Despite not being a full $\mathrm{MI}$ intervention, given the didactic and interactive educational components, fidelity was measured using scales from the Motivational Interviewing Treatment Integrity 3.1.1. Interventionist behavioural counts were measured along with MI Spirit, an average measure of the quality of MI delivery. ${ }^{27}$

\section{Data sources}

The baseline survey included demographic information, housing status and relationship status. Opioid use in the prior 30 days was categorised as: only pharmaceutical opioids whether prescribed to the participant or not, OAT from an approved provider (licit source), heroin and OAT, and OAT no heroin. Route of administration was coded into whether a person had smoked, snorted or injected any opioids ( $96 \%$ reported injecting). Days of opioid use in the prior 30 days was recorded. Protective factors, including whether 'anyone you have regular contact with' had 'overdose education' or 'regularly carry or have quick access to naloxone', were documented. Overdose risk factors included: overdose history; using opioids when no one else was around or behind a locked door; and using opioids within 2 hours of alcohol, sedatives/downers (specific brand names were provided), using another kind of opioid or stimulants including cocaine, methamphetamine or pharmaceutical stimulants.

Healthcare utilisation data from UW Medicine included encounter and billing data from HMC and UWMC, their EDs and affiliated onsite and offsite clinics in the Seattle, Washington, metropolitan area.

The Comprehensive Hospital Abstract Reporting System (CHARS) maintained by the Washington State Department of Health was used for statewide capture of opioid overdose events resulting in inpatient or hospital observation stays. CHARS contains hospital discharge information for inpatient and observation stays derived from billing records for essentially all Washington State community hospitals. Statewide death certificate data were obtained from the Washington State Department of Health.

\section{Outcome measures}

ED visits were defined as an encounter that had at least one charge originating from the ED, regardless of whether it resulted in inpatient admission. Some encounters may have been counted as both an ED visit and an inpatient admission, but each metric was analysed separately. Index encounters were defined as ED visits or inpatient admissions (1) beginning on or before the randomisation date and (2) concluding on or after the randomisation date. ED visits and inpatient admissions were counted separately and included all encounters for any principal diagnosis with admission/visit dates occurring after the discharge date of 


\section{Original article}

Table 1 Participant demographic characteristics, opioid use patterns, overdose history and risk and protective factors

\begin{tabular}{|c|c|c|c|c|c|c|}
\hline \multirow{3}{*}{ Age mean SD } & \multicolumn{2}{|c|}{ Intervention } & \multicolumn{2}{|c|}{ Comparison } & \multirow{2}{*}{\multicolumn{2}{|c|}{$\begin{array}{l}\text { Total } \\
n=241\end{array}$}} \\
\hline & \multicolumn{2}{|l|}{$n=115$} & \multicolumn{2}{|l|}{$\mathrm{n}=126$} & & \\
\hline & 40.2 & 11.5 & 42.3 & 11.5 & 41.3 & 11.5 \\
\hline & $\mathrm{n}$ & $\%$ & $\mathbf{n}$ & $\%$ & $\mathrm{n}$ & $\%$ \\
\hline Female & 32 & 28 & 37 & 29 & 69 & 29 \\
\hline Hispanic & 14 & 12 & 21 & 17 & 35 & 15 \\
\hline Race & & & & & & \\
\hline White & 60 & 53 & 67 & 54 & 127 & 53 \\
\hline Black & 17 & 15 & 14 & 11 & 31 & 13 \\
\hline Asian & 0 & 0 & 2 & 2 & 2 & 1 \\
\hline Hawaiian/Pacific Islander & 0 & 0 & 2 & 2 & 2 & 1 \\
\hline American Indian/Native Alaskan & 6 & 5 & 6 & 5 & 12 & 5 \\
\hline Other & 13 & 11 & 14 & 11 & 27 & 11 \\
\hline More than one race & 18 & 16 & 19 & 15 & 37 & 16 \\
\hline Housing & & & & & & \\
\hline Permanent & 29 & 25 & 44 & 35 & 73 & 30 \\
\hline Impermanent & 19 & 17 & 22 & 18 & 41 & 17 \\
\hline Homeless & 67 & 58 & 59 & 47 & 126 & 53 \\
\hline Education & & & & & & \\
\hline$<$ High School & 38 & 33 & 35 & 28 & 73 & 30 \\
\hline High School & 37 & 32 & 45 & 36 & 82 & 34 \\
\hline$>$ High School & 40 & 35 & 46 & 37 & 86 & 36 \\
\hline Relationship & & & & & & \\
\hline Not in a relationship & 86 & 75 & 83 & 66 & 169 & 71 \\
\hline In a relationship & 28 & 25 & 42 & 34 & 70 & 29 \\
\hline Employment & & & & & & \\
\hline Employed & 9 & 8 & 9 & 7 & 18 & 8 \\
\hline Unemployed & 88 & 77 & 88 & 70 & 176 & 73 \\
\hline Retired & 6 & 5 & 4 & 3 & 10 & 4 \\
\hline Unable to work & 7 & 6 & 22 & 18 & 29 & 12 \\
\hline Other* & 5 & 4 & 2 & 2 & 7 & 3 \\
\hline Opioid type & & & & & & \\
\hline Pharmaceutical only & 18 & 16 & 13 & 10 & 31 & 13 \\
\hline Heroin and no opioid agonist therapy & 67 & 58 & 74 & 59 & 141 & 59 \\
\hline Heroin and opioid agonist therapy & 24 & 21 & 24 & 19 & 48 & 20 \\
\hline Opioid agonist therapy no heroin & 6 & 5 & 15 & 12 & 21 & 9 \\
\hline Smoke/snort/inject opioids & 94 & 82 & 101 & 80 & 195 & 81 \\
\hline $\begin{array}{l}\text { \# of days used opioids past } \\
30 \text { days mean SD }\end{array}$ & 24.3 & 8.4 & 25.1 & 8.8 & 24.7 & 8.6 \\
\hline Know others with overdose education & & & & & & \\
\hline Yes & 45 & 39 & 62 & 49 & 107 & 44 \\
\hline No/do not know & 69 & 60 & 64 & 51 & 133 & 55 \\
\hline Know others who have naloxone & & & & & & \\
\hline Yes & 33 & 29 & 41 & 33 & 74 & 31 \\
\hline No/do not know & 82 & 71 & 85 & 67 & 167 & 69 \\
\hline Opioid overdose history & & & & & & \\
\hline Never overdosed & 48 & 42 & 56 & 44 & 104 & 43 \\
\hline Overdosed, not past 3 months & 48 & 42 & 43 & 34 & 91 & 38 \\
\hline Overdosed past 3 months & 19 & 17 & 27 & 21 & 46 & 19 \\
\hline Used alone past 3 months & 89 & 77 & 100 & 79 & 189 & 78 \\
\hline Always/sometimes use __ within 2 hours of opioids & & & & & & \\
\hline Alcohol & 36 & 31 & 54 & 43 & 90 & 37 \\
\hline Sedatives/downers & 48 & 42 & 61 & 48 & 109 & 45 \\
\hline More than one kind of opioid & 53 & 46 & 54 & 43 & 107 & 44 \\
\hline Uppers: cocaine, methamphetamine, pharmaceutical & 57 & 50 & 72 & 57 & 129 & 54 \\
\hline
\end{tabular}


Table 2 Opioid overdose events and censoring by randomisation status

\begin{tabular}{|c|c|c|c|c|c|c|c|}
\hline \multirow[b]{2}{*}{ Group } & \multirow[b]{2}{*}{ Total N } & \multicolumn{5}{|c|}{ Opioid overdose event status by data source: $\mathbf{n}(\%)$} & \multirow[b]{2}{*}{$\begin{array}{l}\text { Mean days to first } \\
\text { overdose event } \emptyset\end{array}$} \\
\hline & & $\begin{array}{l}\text { First opioid } \\
\text { overdose in UW } \\
\text { Medicine* }\end{array}$ & $\begin{array}{l}\text { First opioid } \\
\text { overdose in } \\
\text { CHARSt }\end{array}$ & $\begin{array}{l}\text { First opioid } \\
\text { overdose fatality } \\
\text { in DOH }\end{array}$ & $\begin{array}{l}\text { Non-overdose } \\
\text { fatality in } \mathrm{DOH} \ddagger\end{array}$ & $\begin{array}{l}\text { No event prior to } \\
\text { censoring at study } \\
\text { end§ }\end{array}$ & \\
\hline Comparison group & 126 & $21(16.7)$ & $6(4.8)$ & $6(4.8)$ & $7(5.6)$ & $86(68.3)$ & 836 \\
\hline Intervention group & 115 & $18(15.7)$ & $4(3.5)$ & $2(1.7)$ & $8(7.0)$ & $83(72.2)$ & 870 \\
\hline Combined & 241 & $39(16.2)$ & $10(4.2)$ & $8(3.3)$ & $15(6.2)$ & $169(70.1)$ & 852 \\
\hline
\end{tabular}

*Local inpatient admission or emergency department visit.

†Statewide hospital discharge for inpatient admission or observation visit.

$\ddagger$ Death certificate data, Department of Health.

§Available follow-up time: 272-1064 days.

IMean is underestimated because the largest observed analysis time is censored.

CHARS, Comprehensive Hospital Abstract Reporting System; UW, University of Washington.

the index visit through 31 December 2015. Index encounters were excluded from outcome encounter counts and excluded from consideration as the first opioid overdose event (described below), because the need for these encounters was evidenced prior to the intervention and hence not properly considered an outcome.

All three administrative data sources (ie, UW Medicine, CHARS and death certificate data) were used jointly to identify the first opioid overdose event occurring after randomisation and discharge from the index encounter and censored at 31 December 2015. Time to the first opioid overdose event was measured from randomisation to the date of the first-occurring qualifying event: (1) UW Medicine ED, inpatient or outpatient encounter for opioid overdose, (2) CHARS inpatient admission or observation stay for opioid overdose or (3) death from opioid overdose. The definitions for an opioid overdose based on ICD-9 (International Classification of Diseases) and ICD-10 codes across datasets are detailed in the online supplement.

\section{Sample size}

Power calculations were based on the estimated annual overdose rate of $20 \%$ for heroin users and $10 \%$ for pharmaceutical opioid users (seen in the ED) and reduction in opioids overdoses of $50 \%$ due to the intervention. The sample size for heroin users to meet these parameters was 219 with 1 year of follow-up for overdose. For pharmaceutical users with an estimated annual overdose rate of $10 \%$, we would require roughly twice the number of subjects or double the follow-up time to have the same number of overdose events.

\section{Data analysis}

Healthcare utilisation outcomes (ie, number of ED visits and number of inpatient admissions) were analysed using negative binomial regression with robust variance estimates and a time at-risk exposure adjustment for available follow-up time. Mean rates per person-year were calculated using these models.

Kaplan-Meier survival function curves were used to depict time from randomisation to the first opioid overdose event, with days of follow-up as the time scale. Death due to causes other than opioid overdose was treated as a censoring event but cannot be considered independent of randomisation assignment. We therefore treated death due to causes other than opioid overdose as a competing risk, using competing risk survival analysis models to analyse time from randomisation to the first opioid overdose event. ${ }^{28}$ The STATA command -stcrreg- (based on the Fine and Gray semiparametric method) was used to produce sub-HRs (SHR). ${ }^{29} 30$

All statistical tests were two tailed, with statistical significance defined as $\mathrm{P} \leq 0.05$. Analyses were performed using Stata/MP V.13.1 for Windows. The study was registered at clinicaltrials. gov (NCT01788306).

\section{RESULTS}

\section{Study enrolment}

Enrollment occurred between 31 January 2013 and 3 April 2015, allowing for at least 272 and up to 1064 days of follow-up, which ended on 31 December 2015. Participant enrolment, allocation and analysis are outlined in the Consolidated Standards of Reporting Trials (CONSORT) diagram in figure 1, as are reasons for study exclusion. Among the 430 assessed for study eligibility, 256 were enrolled and randomised, with 125 allocated to the intervention and 131 to the comparison group.

The study underenrolled compared with the original study design of 500 heroin and 500 pharmaceutical opioid users. The randomisation process appears to have achieved sufficient balance; baseline characteristics in table 1 are comparable (all $\mathrm{P}$ values $>0.05)$. For these analyses only those who provided consent to access secondary data were included: 115 in the

Table 3 Annual local healthcare utilisation (all cause) after study enrolment by randomisation status

\begin{tabular}{|c|c|c|c|c|c|c|c|c|}
\hline \multirow[b]{2}{*}{ Group } & \multicolumn{4}{|c|}{ Emergency department visits* } & \multicolumn{4}{|c|}{ Inpatient admissions } \\
\hline & $\begin{array}{l}\text { Median annual } \\
\text { rate }\end{array}$ & IQR & $\begin{array}{l}\text { Mean annual } \\
\text { ratet }\end{array}$ & $95 \% \mathrm{Cl}$ & $\begin{array}{l}\text { Median annual } \\
\text { rate }\end{array}$ & IQR & $\begin{array}{l}\text { Mean annual } \\
\text { ratet }\end{array}$ & $95 \% \mathrm{Cl}$ \\
\hline Comparison group & 2.72 & 5.49 & 4.85 & 3.96 to 5.93 & 0.43 & 1.50 & 1.05 & 0.80 to 1.39 \\
\hline Intervention group & 2.42 & 7.35 & 4.96 & 4.04 to 6.10 & 0.39 & 1.80 & 1.29 & 0.95 to 1.76 \\
\hline Combined & 2.57 & 6.93 & 4.90 & 4.25 to 5.66 & 0.41 & 1.52 & 1.17 & 0.95 to 1.44 \\
\hline
\end{tabular}

Note: $r$ ate differences between the intervention and comparison groups were not statistically significant.

${ }^{*}$ Regardless of discharge status (may have resulted in inpatient admission).

tMean rate per person-year calculated using negative binomial regression with time at-risk exposure variable for available follow-up time (272-1064 days, censored at death or 31 December 2015). 


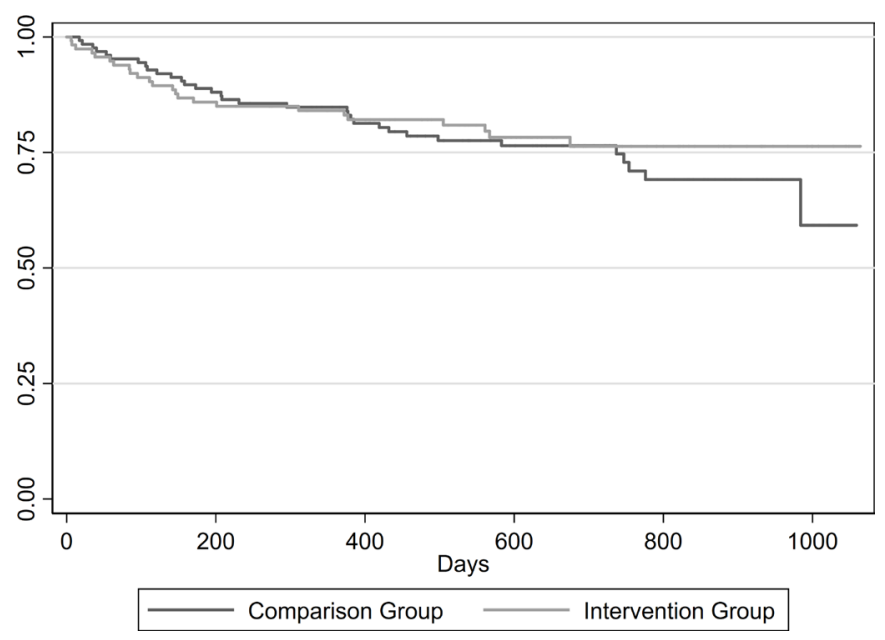

Figure 2 Time to the first opioid overdose event (encounter or death) $(n=241)$.

intervention arm and 126 in the comparison group, a combined total of 241. The care setting in which participants were recruited included ED $(n=149)$, respite care $(n=37)$ and hospital inpatient $(n=55)$ and did not differ significantly by study assignment. We identified potential subjects as quickly as possible and among those enrolled in respite care $89 \%$ had their baseline assessment completed within 3 days of starting the assessment (maximum 5 days) and among inpatients $98 \%$ had the assessment completed within 2 days (maximum 3 ).

\section{Characteristics of intervention and comparison groups}

The majority of participants were male, white, non-Hispanic, homeless, not in a relationship, unemployed, used heroin and not in OAT and used opioids by routes of ingestion including smoking, snorting or injecting (table 1). The mean number of days that opioids were used in the past 30 days was 24.7 (8.6 SD) with a median of 30 . Protective factors for overdose were reported by a minority of participants including others in their life having overdose education or possessing naloxone. The majority had a history of an opioid overdose, $19 \%$ in the prior 3 months and $38 \%$ sometime prior to the most recent 3 months. The vast majority, $78 \%$, reported using opioids alone sometimes or always in the prior 3 months, and many reported using a range of other substances within 2 hours of using opioids. A substantial minority reported having had prior overdose education and knowing others who had naloxone.

\section{MI fidelity}

MI Spirit was calculated on 61 intervention sessions (out of 76 recordings), with an average score of 4.23 (out of 5). Recorded length across the 76 sessions for the overdose education and brief behaviour change counselling content averaged $27 \mathrm{~min}$, with length ranging from $10 \mathrm{~min}$ to $55 \mathrm{~min}$.

\section{Overdose events by intervention assignment}

Opioid overdose events and censoring by randomisation status are presented in table 2 and indicate that $23.7 \%$ of participants had at least one overdose event of some type, $6.2 \%$ had a non-overdose fatality and $70.1 \%$ had no observed event prior to censoring at the end of study follow-up.

\section{Healthcare utilisation by intervention assignment}

The majority $(55 \%)$ of all participants had a hospital admission during the follow-up period with an average annual rate of 1.17 (95\% CI 0.95 to 1.44 ) visits and with no significant difference between the intervention and comparison groups (table 3). A substantial majority $(85 \%)$ of all participants had a subsequent ED visit during the follow-up period, with an average annual rate of 4.90 (95\% CI 4.25 to 5.66) visits and with no significant difference by intervention assignment.

\section{Time to first overdose by intervention assignment}

In the competing risk regression analysis, the difference in the time to first overdose event was not significantly lower for the intervention group relative to the comparison group (SHR: 0.83; $95 \%$ CI 0.49 to 1.40 ). These data are presented as a survival curve in figure 2.

\section{DISCUSSION}

In this study of patients at high risk for opioid overdose presenting for or soon after emergency care, an overdose prevention intervention was found to have no statistically significant impact on subsequent overdoses, either positive or negative. This null finding is perhaps not surprising given the medical and social acuity of the population in terms of homelessness, drug use and other health and social issues. A brief, one-time intervention in acute care settings or subsequent to receiving acute care may not be sufficient to reduce serious overdose events. The multipart intervention was likely more intensive and time consuming than most overdose education and naloxone distribution programme in community or medical settings, although there is great heterogeneity in these interventions.

Population-based studies have found decreased mortality rates associated with distributing naloxone to illicit drug users. $^{25}$ Estimates of the lifetime impact of naloxone distribution to individual heroin users are modest, a $6 \%$ mortality reduction. ${ }^{31}$ Naloxone distribution programmes provide clear life-saving benefits; however, they are also insufficient to substantially address opioid overdose alone. Brief interventions in the ED have shown modest benefits in decreasing opioid-related risk behaviours and drug use; however, these studies were of those with recent prescription opioid misuse and used a much broader definition of overdose that was not limited to opioids. ${ }^{13} 14$ We did not use self-report of overdose as an outcome as follow-up rates at each time point were less than $50 \%$, perhaps due to the high levels of housing impermanence. We chose to enrol a high-acuity population, despite knowing that this might lessen the impact of the intervention and lower follow-up rates as we felt it was important to enrol a population representative of that seen in the care settings. It is possible that the informational flyer provided to the comparison group combined with the substantial increases in takehome naloxone within the community was sufficient to reduce any differential effect of the intervention.

The statistical power to detect differences was limited by the sample size and given the modest, though significant, impact of naloxone distribution found in other studies likely was an important limitation in our ability to find any potential impact of our intervention. We attempted to address this by adding measures of non-fatal overdose as an outcome because studies with an outcome of fatal overdose require sample sizes of many thousands, ${ }^{26}$ but we could only capture overdose events that resulted in an outpatient visit, ED or hospital 
admission. Naloxone distribution studies are further limited in that naloxone distributed to one person is often administered to another, potentially underestimating the total impact of the intervention. We did not account for heroin users being on OAT in our sampling design, which is a complicating issue in that research indicates being on OAT reduces fatal overdose occurrence, potentially reducing the overdose event rate and the statistical power to detect any differences. ${ }^{32} \mathrm{~A}$ subanalysis of the time to overdose event adjusting for opioid-use type did not significantly impact results (data not shown).

Study recruitment was challenging given the care settings and the acuity of the population, acute care settings are very hectic and complicated the logistics identifying, approaching and enrolling subjects in a study. Medical providers were reoriented to the study multiple times in order to reinforce that pharmaceutical opioid users, including those with a prescription for the medications, were indeed eligible for the study. There were also challenges in identifying pharmaceutical users due to very limited use of and access to Washington State's prescription drug monitoring programme (PDMP) by medical providers at the time of the study. Study staff were precluded from accessing the PDMP for research purposes per state law.

In addition to overdose education, counselling and naloxone, other more robust interventions such as direct referral and provision of housing and OAT medications may be necessary to have a clinically significant impact on opioid overdoses for this highacuity population served in acute care settings. The ED is a challenging setting for delivering an intervention logistically, in terms of timing and space constraints, and due to the medical state of patients. The most common reasons for refusing the screening interview $(\mathrm{n}=510)$, among those approached $(\mathrm{n}=936)$ were 'not interested' (49\%), 'not feeling well' (39\%) and 'no time' (8\%). Only a small proportion of those enrolled in the study were seen for an opioid overdose (12\%). Recent findings suggest that brief overdose and naloxone training is sufficient, and it appears that a population level mortality benefit is associated with higher rates of naloxone distribution in a community. ${ }^{253-40}$ Therefore, ED overdose prevention interventions might reasonably be limited to brief education with the direct provision of take-home naloxone. Future research on the impact of a more modest intervention with a larger number of acute care participants may be worthwhile.

\section{What is already known on the subject}

Opioid overdoses are increasing rapidly.

- Overdose education and take-home naloxone decrease population rates of overdose.

\section{What this study adds}

- A brief behavioural-educational intervention combined with an offer of naloxone did not reduce opioid overdose events or health care utilisation among a high-acuity population seen in postacute care.

- Patients seen in acute care settings at elevated risk for overdose had very high rates of subsequent emergency department visits and hospitalisations and warrant more intensive interventions.

- Clinical trials of emergent issues may be impacted by rapid changes in the public health and healthcare environments.

\section{Author affiliations}

${ }^{1}$ Alcohol \& Drug Abuse Institute and Department of Health Services, School of Public Health University of Washington, Seattle, Washington, USA

${ }^{2}$ Substance Use Research, San Francisco Department of Public Health, San Francisco, California, USA

${ }^{3}$ Department of Medicine, University of California San Francisco, San Francisco,

California, USA

${ }^{4}$ Department of Medicine, University of Washington, Seattle, Washington, USA ${ }^{5}$ Department of Health Services, School of Public Health and Harborview Injury Prevention and Research Center, University of Washington, Seattle, Washington, USA

${ }^{6}$ Institute for Work and Health, Toronto, Ontario, Canada

${ }^{7}$ Department of Psychiatry \& Behavioral Sciences, University of Washington, Seattle, Washington, USA

${ }^{8}$ Alcohol \& Drug Abuse Institute and Department of Epidemiology, School of Public Health, University of Washington, Seattle, Washington, USA

${ }^{9}$ Department of Emergency Medicine, University of Washington, Seattle, Washington, USA

${ }^{10}$ Oregon Health \& Science University/Portland State University School of Public Health, Oregon Health \& Science University, Portland, Oregon, USA

${ }^{11}$ Alcohol \& Drug Abuse Institute and Department of Psychiatry \& Behavioral Sciences, University of Washington, Seattle, Washington, USA

Acknowledgements We would like to thank: Barbara Burke, Leslie Enzian, Shira Hasson-Schiff, Sheree Miller, Susan Stern, Steve Mitchell, MelissaPhares, Jeff Purcell, Harborview Emergency Department, Harborview Adult Medicine Clinic, Harborview Respite Program at Jefferson Terrace (Edward Thomas House), Data Safety Monitoring Oversight Committee and Administrative Support Staff at the UW Alcohol and Drug Abuse Institute.

Contributors CJB-G, POC, JOM, CD, DMD, NDY and ASF substantially contributed to the conception and design of the study. CJB-G, ASF, JMS and LKW also contributed to acquiring data. All authors were involved in analysis and interpretation of the data, drafting and revising the article and provided final approval of the submitted manuscript.

Funding Research reported in this publication was supported by the National Institute on Drug Abuse of the National Institutes of Health under award number 1R01A030351. Study data were collected and managed using REDCap electronic data capture tools hosted at the Institute of Translational Health Sciences. REDCap at ITHS is supported by the National Center for Advancing Translational Sciences of the National Institutes of Health under Award Number UL1 TR002319.

Disclaimer The content is solely the responsibility of the authors and does not necessarily represent the official views of the National Institutes of Health.

Competing interests POC has previously directed National Institutes of Health-funded trials that have received donated study medications from Alkermes (2014-2015) and Gilead (2015-2017).

Ethics approval University of Washington Human Subjects Division and Washington State Institutional Review Board.

Provenance and peer review Not commissioned; externally peer reviewed.

(c) Article author(s) (or their employer(s) unless otherwise stated in the text of the article) 2019. All rights reserved. No commercial use is permitted unless otherwise expressly granted.

\section{REFERENCES}

1 Jones CM, Mack KA, Paulozzi LJ, et al. Pharmaceutical overdose deaths, United States, 2010. JAMA 2013:309:657-9.

2 Rudd RA, Seth P, David F, et al. Increases in drug and opioid-involved overdose deaths United States, 2010-2015. MMWR Morb Mortal Wkly Rep 2016;65:1445-52.

3 Weiss AJ, Elixhauser A, Barrett ML, et al. Opioid-related inpatient stays and emergency department visits by state, 2009-2014. HCUP Stat Br 2014:219:1-15.

4 McGregor C, Darke S, Ali R, et al. Experience of non-fatal overdose among heroin users in Adelaide, Australia: circumstances and risk perceptions. Addiction 1998;93:701-11.

5 Baca CT, Grant KJ. Take-home naloxone to reduce heroin death. Addiction 2005;100:1823-31.

6 Fairbairn N, Coffin PO, Walley AY. Naloxone for heroin, prescription opioid, and illicitly made fentanyl overdoses: challenges and innovations responding to a dynamic epidemic. Int J Drug Policy 2017;46:172-9.

7 Davidson PJ, Ochoa KC, Hahn JA, et al. Witnessing heroin-related overdoses: the experiences of young injectors in San Francisco. Addiction 2002;97:1511-6.

8 Strang J, Powis B, Best D, et al. Preventing opiate overdose fatalities with takehome naloxone: pre-launch study of possible impact and acceptability. Addiction 1999:94:199-204.

9 Miller WR, William R, Rollnick S. Motivational interviewing : preparing people for change: Guilford Press, 2002. 
10 Whitlock EP, Polen MR, Green CA, et al. Behavioral counseling interventions in primary care to reduce risky/harmful alcohol use by adults: a summary of the evidence for the U.S. Preventive Services Task Force. Ann Intern Med 2004;140:557.

11 Estee S, Wickizer T, He L, et al. Evaluation of the Washington state screening, brief intervention, and referral to treatment project. Med Care 2010;48:18-24.

12 Gentilello LM, Rivara FP, Donovan DM, et al. Alcohol interventions in a trauma center as a means of reducing the risk of injury recurrence. Ann Surg 1999;230:473-80. discussion 480-3.

13 Bohnert AS, Bonar EE, Cunningham R, et al. A pilot randomized clinical trial of an intervention to reduce overdose risk behaviors among emergency department patients at risk for prescription opioid overdose. Drug Alcohol Depend 2016;163:40-7.

14 Blow FC, Walton MA, Bohnert ASB, et al. A randomized controlled trial of brief interventions to reduce drug use among adults in a low-income urban emergency department: the HealthiER You study. Addiction 2017;112:1395-405.

15 Fisher JD, Fisher WA, Amico KR, et al. An information-motivation-behavioral skills model of adherence to antiretroviral therapy. Heal Psychology 2006;25:462-73.

16 Maxwell S, Bigg D, Stanczykiewicz K, et al. Prescribing naloxone to actively injecting heroin users. J Addict Dis 2006;25:89-96.

17 Sporer KA, Kral AH. Prescription naloxone: a novel approach to heroin overdose prevention. Ann Emerg Med 2007;49:172-7.

18 Wheeler E, Jones TS, Gilbert MK, et al. Opioid overdose prevention programs providing naloxone to laypersons - United States, 2014. MMWR Morb Mortal Wkly Rep 2015;64:631-5.

19 WHO. Community management of opioid overdose: Springer Reference, 2014.

20 SAMHSA. Opioid Overdose TOOLKIT facts for community members five essential steps for first reponders information for prescribers safety advice for patients \& family members recovering from opioid overdose. : HHS Publ, 2013:4742: 13. http://www. store.samhsa.gov

21 Doe-Simkins M, Walley AY, Epstein A, et al. Saved by the nose: bystander-administered intranasal naloxone hydrochloride for opioid overdose. Am J Public Health 2009;99:788-91.

22 Green TC, Heimer R, Grau LE. Distinguishing signs of opioid overdose and indication for naloxone: an evaluation of six overdose training and naloxone distribution programs in the United States. Addiction 2008;103:979-89.

23 Lagu T, Anderson BJ, Stein M. Overdoses among friends: drug users are willing to administer naloxone to others. J Subst Abuse Treat 2006;30:129-33.

24 Seal KH, Downing M, Kral AH, et al. Attitudes about prescribing take-home naloxone to injection drug users for the management of heroin overdose: a survey of streetrecruited injectors in the San Francisco Bay Area. J Urban Health 2003:80:291-301.

25 Walley AY, Xuan Z, Hackman HH, et al. Opioid overdose rates and implementation of overdose education and nasal naloxone distribution in Massachusetts: interrupted time series analysis. BMJ 2013;346:f174.
26 Strang J, Bird SM, Parmar MK. Take-home emergency naloxone to prevent heroin overdose deaths after prison release: rationale and practicalities for the N-ALIVE randomized trial. J Urban Health 2013;90:983-96.

27 Moyers TB, Martin T, Manuel JK, et al. Revised global scales: Motivational Interviewing Treatment Integrity 3.1.1 (MITI 3.1.1). 2010 https://casaa.unm.edu/download/miti3_ 1.pdf (accessed 25 Oct 2017).

28 Pintilie M. Competing risks : a practical perspective. West Sussex, England: John Wiley \& Sons, 2006. https://books.google.com/books?hl=en\&lr=\&id=ZRh2glU6isC\&oi=fnd\&pg =PR7\&dq=Pintilie+M.+Competing +Risks:+A+Practical+ Perspective.+West+Sussex, + England:+Wiley\%3B+2006.\&ots=NdLT548oNj\&

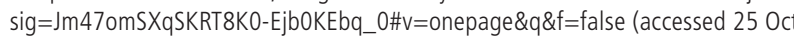
2017).

29 Cleves MA, Gould WW, Gutierrez RG, et al. An introduction to survival analysis using Stata, 2008

30 Fine JP, Gray RJ. A proportional hazards model for the subdistribution of a competing risk. J Am Stat Assoc 1999:94:496-509.

31 Coffin PO, Sullivan SD. Cost-effectiveness of distributing naloxone to heroin users for lay overdose reversal. Ann Intern Med 2013;158:1-9.

32 Pierce M, Bird SM, Hickman M, et al. Impact of treatment for opioid dependence on fatal drug-related poisoning: a national cohort study in England. Addiction 2016;111:298-308.

33 Larimer ME, Malone DK, Garner MD, et al. Health care and public service use and costs before and after provision of housing for chronically homeless persons with severe alcohol problems. JAMA 2009;301:1349-57.

34 Banta-Green CJ, Coffin PO. Commentary on Pierce et al. (2016): raising the bar of addiction treatment--first do no harm. Addiction 2016;111:309-10.

35 Polsky D, Glick HA, Yang J, et al. Cost-effectiveness of extended buprenorphinenaloxone treatment for opioid-dependent youth: data from a randomized trial. Addiction 2010;105:1616-24.

36 Hser YI, Evans E, Huang D, et al. Long-term outcomes after randomization to buprenorphine/naloxone versus methadone in a multi-site trial. Addiction 2016;111:695-705.

37 D'Onofrio G, O'Connor PG, Pantalon MV, et al. Emergency department-initiated buprenorphine/naloxone treatment for opioid dependence. JAMA 2015;313:1636.

38 D'Onofrio G, Chawarski MC, O'Connor PG, et al. Emergency department-initiated buprenorphine for opioid dependence with continuation in primary care: outcomes during and after intervention. J Gen Intern Med 2017;32:660-6.

39 Kourounis G, Richards BD, Kyprianou E, et al. Opioid substitution therapy: lowering the treatment thresholds. Drug Alcohol Depend 2016;161:1-8.

40 Behar $E$, Santos GM, Wheeler $E$, et al. Brief overdose education is sufficient for naloxone distribution to opioid users. Drug Alcohol Depend 2015;148:209-12. 